\title{
Can we recognize people based on their body-alone? The roles of body motion and whole person context.
}

\author{
Noa Simhi ${ }^{1}$ and Galit Yovel ${ }^{1,2}$ \\ ${ }^{1}$ The School of Psychological Sciences and ${ }^{2}$ The Sagol School of Neuroscience, Tel-Aviv University \\ Correspondence regarding this manuscript should be addressed to:
}

Galit Yovel and Noa Simhi: gality@post.tau.ac.il, simhinoa@gmail.com

\begin{abstract}
While most studies on person recognition examine the face alone, recent studies have shown evidence for the contribution of the body and gait to person recognition beyond the face. Nevertheless, little is known on whether person recognition can be performed based on the body alone. In this study, we examined two sources of information that may enhance body-based person recognition: body motion and whole person context. Body motion has been shown to contribute to person recognition especially when facial information is unclear. Additionally, generating whole person context, by attaching faceless heads to bodies, has been shown to activate face processing mechanisms and may therefore enhance body-based person recognition. To assess body-based person recognition, participants performed a sequential matching task in which they studied a video of a person walking followed by a headless image of the same or different identity. The role of body motion was examined by comparing recognition
\end{abstract}


from dynamic vs. static headless bodies. The role of whole person context was examined by comparing bodies with and without faceless heads. Our findings show that body motion contributed to body-based person recognition, and person recognition from the body alone was better in dynamic vs. static displays. In addition, whole person context contributed to body-based person recognition when recognition was performed in static displays. Overall these findings show that recognizing people based on their body alone is challenging but can be performed under certain circumstances that enhance the processing of the body when seeing the whole person. 


\section{Introduction}

Face and body processing are similar in many ways - there are specialized face processing areas (Kanwisher, McDermott, \& Chun, 1997; Kanwisher \& Yovel, 2006) as well as body processing areas (Downing, Jiang, Shuman, \& Kanwisher, 2001; Peelen \& Downing, 2005) in the brain. Bodies can be detected as quickly as faces (Bindemann, Scheepers, Ferguson, \& Burton, 2010). It has been shown that both the body and the face contribute to person recognition (O’Toole et al., 2011; Simhi \& Yovel, 2016), and also that person recognition can be based mostly on the body even when participants are unaware of doing so (Rice, Phillips, Natu, An, \& O’Toole, 2013). Nonetheless, less is known on whether person recognition can be performed based on the body alone, and which factors are important for this process.

While person recognition from the face alone - from profile pictures, passport photos etc. is common, there is less evidence on whether person recognition can be performed based on the body alone. Most studies that examined the role of bodies in person recognition have examined their contribution to the recognition of the whole person beyond the face. Several of these studies have shown that the contribution of the body beyond the face is primarily found when people are seen in motion rather than in static images (O'Toole et al., 2011; Simhi \& Yovel, 2016) and that information from moving bodies is particularly useful when seeing people at a distance (Hahn, O’Toole, \& Phillips, 2015; Simhi \& Yovel, 2019), when facial features are unclear and in many cases are not informative enough for identification (see Yovel \& O’Toole, 2016 for a review). 
Some of the aforementioned studies also examined person recognition based on the body alone. For example, O’Toole et al., 2011 included a condition in which faces were blurred in order to create a body only recognition condition. In this study it was found that person recognition from the body alone was relatively poor (as was the case in Hahn et al., 2015 which included a similar condition as well), but was improved by presentation of the body in video, indicating that motion contributes to body-based person recognition. In this sequential matching study however both stimuli were either presented in static images or in videos. Thus, it is was not possible to differentiate between two possible contributions of motion to person recognition - form-frommotion processes and dynamic identity signatures (Simhi \& Yovel, 2016; see also O’Toole, Roark, \& Abdi, 2002 for a description of these in the context for faces, and Yovel \& O'Toole, 2016 for a review explaining their possible role in whole person recognition). The contribution of motion to person recognition through form-from-motion processes, indicates that seeing a person in motion may improve the representation of their body form. Motion can also contribute to person recognition through dynamic identity signatures - these are idiosyncratic motion patterns of an individual which may be used to recognize them (a limp, or hand swing for example). Simhi \& Yovel (2016) used an experimental paradigm that enabled dissociating the contributions of formfrom-motion processes and dynamic identity signatures in whole person recognition. It was found that form-from-motion processes contributed to recognition while dynamic identity signatures did not. In later studies, it was shown that familiarity (Simhi \& Yovel, 2017) and distinctiveness of gait (Simhi \& Yovel, 2019) did influence the contribution of dynamic identity signatures to whole person recognition. In Simhi \& Yovel, 2016, 2017, 2019, however, only whole 
person or face only stimuli were shown. It thus remains to be seen if dynamic identity signatures from body motion contribute to person recognition based on the body alone.

Another important factor which may influence body-based person recognition is whole person context. Recent research has highlighted that whole person context significantly affects different aspects of person perception. It has been shown that seeing a faceless head in body context activates face processing mechanism (Brandman \& Yovel, 2010, 2012; Cox, Meyers, \& Sinha, 2004). Furthermore, seeing an uninformative, approaching body along with a face improves face-based person recognition (Pilz, Vuong, Bülthoff, \& Thornton, 2011). Nonetheless, the contribution of whole person context to body-based person recognition has yet to be examined. This is especially important because studies examining body-based person recognition have used highly variable methods to create body only stimuli. Many studies blur the face to different levels (Hahn et al., 2015; O'Toole et al., 2011), some studies replace the head with a large hat (Robbins \& Coltheart, 2015) and some even include the external facial features (hair/face outline) as part of the body, removing internal facial features alone (Rice et al., 2013). Such methods may create the perception of whole person context since the general shape of the head can be seen, or its presence can be inferred. It is possible that body-based recognition in such conditions is influenced by the remaining head context, and that recognition would be impaired if the head were completely removed rather than obscured. Thus, to fully understand which factors contribute to person recognition based on the body alone it is important to explore whether the activation of face processing mechanisms, as a result of having whole person context, contributes to body-based person recognition. 
Experiment 1 was designed to examine the contribution of dynamic identity signatures and whole person context in body-based person recognition. In order to assess the contribution of body motion, and specifically dynamic identity signatures, to body-based person recognition, we presented participants with different identities in videos and then compared recognition of these identities in videos of the body alone to recognition from multi-static images of the body alone (see Figure 1). In order to assess the additional contribution of whole person context to bodybased dynamic person recognition, we compared recognition from the videos of the body alone (which did not have whole person context) to recognition from videos where the heads were blurred (and thus whole person context was available, since the general shape of the head was visible, but only the body could be used for person recognition) (see Figure 1).

\section{Experiment 1: The effect of dynamic identity signatures and whole person context on body- based person recognition}

\subsection{Methods}

\subsubsection{Participants}

45 participants took part in this experiment (mean age $=22.44, \mathrm{SD}=2.44,40$ female). Participants were allocated to three different between participant conditions, each containing 15 participants. Participants were recruited at Tel Aviv University and participated in the study for either course credit or payment. All participants had normal or corrected to normal vision and gave their informed consent to participate in the study by signing the appropriate consent form approved by the Tel Aviv University ethics committee. 


\subsubsection{Stimuli}

Study stimuli in this experiment were the same as those in Simhi \& Yovel, 2016. The stimuli were adapted from the Database of Moving Faces and People (O'Toole et al., 2005). There were forty-five identities selected for the experiment (15 men), and for each identity there were two different stimuli, filmed on different occasions (up to six months apart), which were used for the experiment.

The first stimulus depicting each identity was used as a study stimulus in each stimulus pair. This stimulus was a video depicting the identity walking towards the participant in a naturally lit corridor in a natural pace. The video was cut at the point where the shoes were no longer visible, so that the identities' full body was visible in every frame of the video. Since the identities walked at a natural pace, this resulted in videos of different lengths (2-7 secs) for each identity (with a mean length of 5.6 secs).

Test stimuli in the experiment consisted of three different types and differed between conditions (see Figure 1); all test stimuli were cut so that they were 4 secs in length, and ended when the identities' shoes were no longer visible (as a result of this, and the differences in walking pace, each identity appeared at a slightly different distance in the first frame of the video). The stimuli were created with a video editing software - Lightworks (EditShare EMEA). The three test stimuli were:

I. Dynamic bodies with whole person context - In order to create these videos, the stimuli were edited using Lightworks so that the head of each identity was blurred. This created 
stimuli in which the head shape was clearly visible, along with some external cues (for example, the color of the hair could sometimes be inferred in these videos), however no facial features were clearly visible. In this way we created stimuli in which the main information available for person recognition was the moving body, but there was still whole person context due to the shape of the head being visible in these stimuli, in contrast to the videos of the body alone.

II. Dynamic bodies without whole person context - These stimuli were created by covering the head in each video with a rectangle that was colored in the same shade as the wall behind the identity, in order to attract minimal attention to the rectangle itself, and create the appearance of a headless body. The rectangle was moved at a minimum, when needed in order to ensure that the face and neck were covered for the duration of the video.

III. Multi-static body without whole person context - These stimuli were created by extracting four static images from the videos of the body without whole person context, for each identity. One image was extracted from each second of the video and the final frame that was extracted was the final frame in the video (in cases where the identity was mid-stride the closest frame was extracted so that the identity appeared in a relatively static position). In order to avoid illusory motion effects, the images were presented in a pseudo-random order, beginning with the first image (with the identity furthest away from the participant), then the third image extracted followed by the second image 
extracted and finally the last image in the series (with the identity closest to the participant). Each static image was presented for $1 \mathrm{sec}$ with $0.1 \mathrm{sec}$ between images.

Thus, comparison between person recognition from videos vs. multi-static images of the headless body would reveal whether dynamic identity signatures from body motion contribute to body-based person recognition. Comparison between person recognition in videos with and without a blurred, faceless head would reveal whether whole person context contributes to person recognition. Examples of these test stimuli can be seen in Figure 1.

\subsubsection{Design}

The experiment consisted of a sequential matching task in which stimuli were presented in pairs and the participants were asked to determine if each pair of stimuli depicted the same identity or not. Care was taken to create non-trivial mismatches in order to ensure that the task was sufficiently difficult, by pairing mismatches according to the similarity between identities (in the same manner described in Simhi \& Yovel, 2016). The design was created using pseudorandom pairing of identities within groups of identities of the same sex, with roughly similar appearance, as determined by two independent observers with the assistance of a third observer

in cases of disagreement. After pseudo-random pairing, the same design was used for all participants, in all test conditions.

The study stimulus in each pair of stimuli, in all three conditions, consisted of a video of a person approaching the participant. Participants were allocated to one of the three test 
conditions (see Figure 1). Half of the trials in each experiment were match trials and half were mismatch trials.

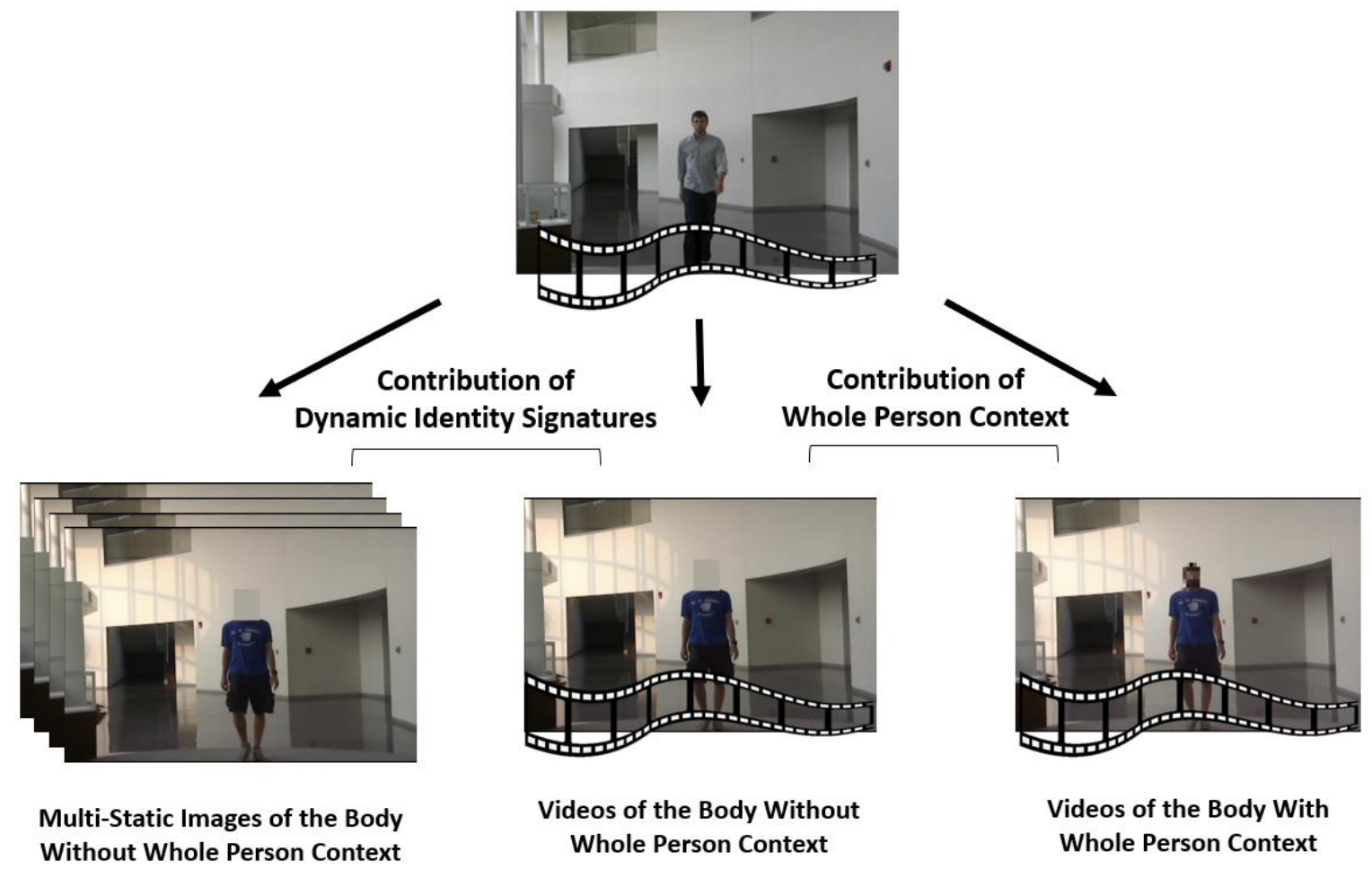

Figure 1. The design of Experiment 1. In a sequential matching task, a video of a whole person was followed by the same or a different identity shown in three different, between participant, conditions. To assess the contribution of dynamic identity signatures, recognition from videos of the headless body (without whole person context) (center) was compared to recognition from multi-static images taken from the videos (left). To assess the contribution of whole person context, recognition from videos of the headless body (without whole person context) (center) was compared to recognition from videos of bodies with whole person context (created by blurring the faces in the videos, to render facial features unavailable while the head shape remained visible) (right). 


\subsubsection{Apparatus and Procedure}

The experiment was presented on a Samsung SyncMaster SA950, Full HD, LED monitor using MATLAB and the Psychophysics Toolbox extension (Brainard, 1997; Kleiner et al., 2007; Pelli, 1997). Participants were seated at a comfortable distance of approximately $60 \mathrm{~cm}$ from the monitor.

In each trial in the experiment, participants viewed two stimuli, sequentially, and determined if the same identity appeared in both stimuli. Participants completed a practice trial before the experiment began, in which the task was explained to them and they could see an example of the experimental procedure. In each of the 44 trials that followed, participants first saw a fixation point for 0.75 secs, followed by a study video. At the end of the study video participants saw 0.5 secs of blank screen followed by another fixation point which was presented for 0.75 secs and was followed by the test stimulus, according to the test condition. Each test stimulus was presented at test for $4 \mathrm{sec}$ (along with three $0.1 \mathrm{sec}$ blank screen between stimulus intervals in the case of the multi-static stimulus presentation). Participants could make their recognition response only after the stimulus (be it a video or a series of multi-static images) disappeared from the screen. After the response a confidence scale of 1-5 appeared on the screen and participants were asked to indicate how confident they were in their recognition decision. After this response a new trial began.

Every 10 trials participants took a break of 5 secs at least. 


\subsubsection{Data Analysis}

In order to analyze the participants' sensitivity and specificity in person recognition in this study, we plotted receiver operating characteristic (ROC) curves, and calculated the area under the curve $(A \cup C)$ per participant. We then compared the AUC between the experimental conditions, where a greater AUC indicates greater discrimination ability. In order to calculate the ROC curves, we used the confidence ratings, which were scaled to a 1-5 scale for each participant. Since confidence ratings were performed after the recognition response, which could be 'yes' or 'no', we multiplied all confidence ratings after a 'no' recognition response by -1 , in order to achieve an informative $(-5)-(+5)$ confidence scale which could be used for the ROC creation (Pashler, 2002).

In addition, similarly to O'Toole et al., 2011 and Simhi \& Yovel, 2016, we complemented the AUC analysis with an analysis of the performance level using the measure of sensitivity, $d^{\prime}$. For the purpose of $d^{\prime}$ measurement we calculated the number of match trials in which participants responded 'yes', which was their hit rate, and the number of mismatch trials in which participants responded 'yes', which was their false alarm rate.

We also looked at the participants' response bias, as measured by the criterion, $\mathrm{C}$, the participants' confidence ratings on correct trials, and the response times (RT) on correct trials in the different conditions.

Trials with RTs longer than 10 secs were removed from all analyses. Statistical analysis was performed using JASP (Version 0.10.0) and RStudio. 


\subsection{Results}

In order to assess the contribution of dynamic identity signatures and whole person context to body-based person recognition we first examined the ROC curves in all three recognition conditions in Experiment 1. These results can be seen in Figure $2(A)$. From the ROC curves we can see that dynamic identity signatures from body motion appear to strongly contribute to body-based person recognition, while whole person context has little additional contribution beyond the contribution of dynamic identity signatures.

In order to statistically assess these effects, we analyzed the AUC in the three experimental conditions. These results can be seen in Figure 2 (B) and Table 1. A one-way ANOVA examining the three recognition conditions revealed a significant effect of condition $(F(2,42)=12.03, p<$ $\left..001, \eta_{p}^{2}=.36\right)$. In order to examine the contribution of dynamic identity signatures to discriminability in body-based person recognition we conducted a Student's t-test between the AUC values when recognizing people from multi-static images of the body alone as compared to videos of the body alone. We found that dynamic identity signatures indeed significantly contributed to body-based person recognition $(t(28)=-3.38, p=.002$, Cohen's $d=-1.23)$.

In order to assess if whole person context contributes to body-based person recognition beyond dynamic identity signatures, we conducted a Student's t-test on the AUC values comparing recognition from videos of the body with and without whole person context. We found that whole person context did not contribute beyond dynamic identity signatures to discriminability in body-based person recognition $(t(28)=.92, p=.36$, Cohen's $d=.34$ ). 


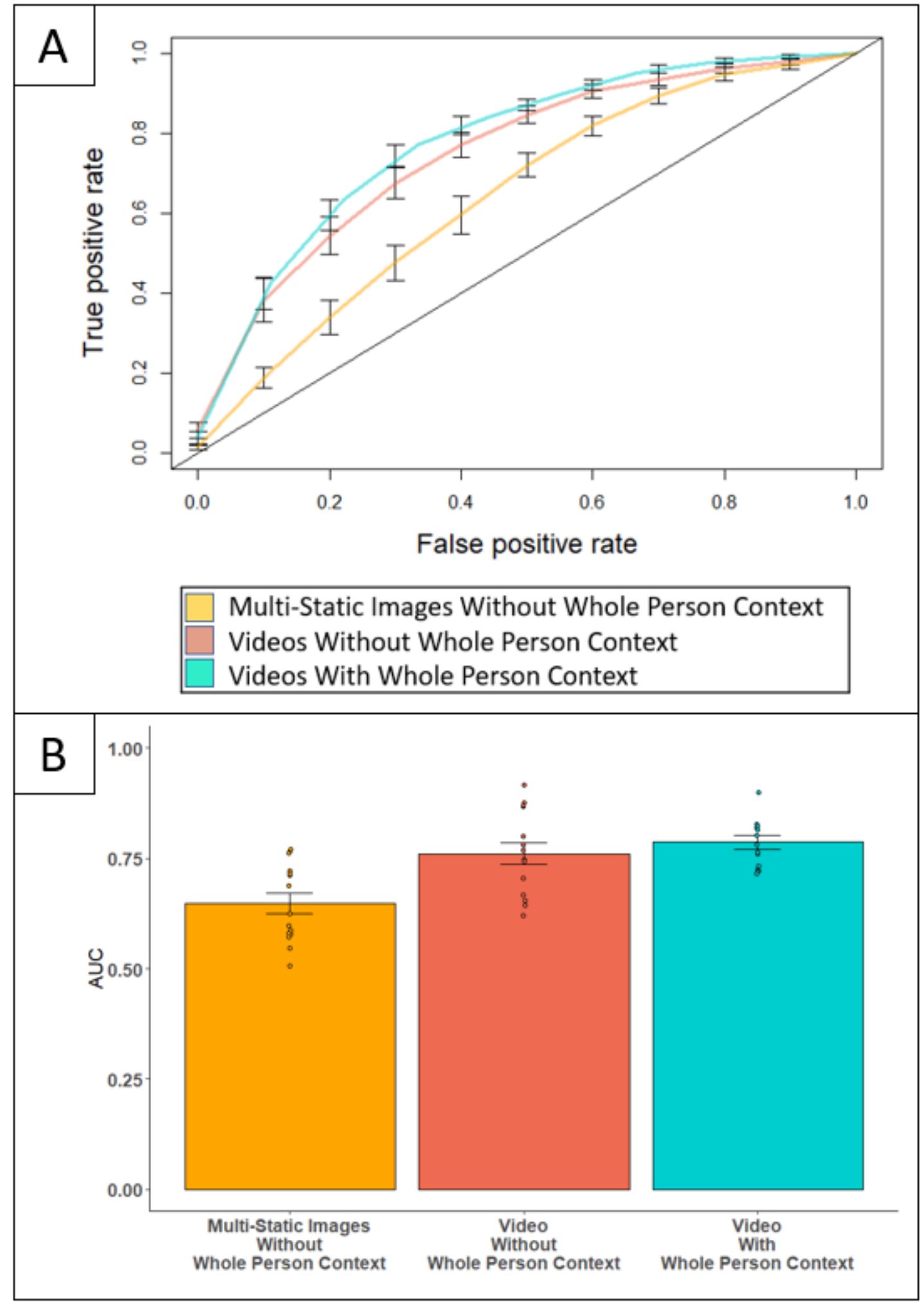


Figure 2. The ROC curve (A) and AUC (B) in the three different conditions in Experiment 1. These findings indicate that dynamic identity signatures from body motion contributed to body-based person recognition, whereas whole person context had no effect. Error bars represent SEM for both panels, and points in Panel B mark the AUC scores of different participants within each test condition.

In order to compare the current findings to previous studies that used d' (O'Toole et al., 2011 and Simhi \& Yovel, 2016), we also assessed discrimination ability using d'. A one-way ANOVA examining the three recognition conditions revealed a significant effect of condition $\left(F(2,42)=7.65, p=.001, \eta_{p}^{2}=.27\right)$. A Student's t-test on $d^{\prime}$ values revealed that there was a contribution of dynamic identity signatures from body motion to body-based person recognition $(t(28)=-2.79, p=.009$, Cohen's $d=-1.02)$ but no contribution of whole person context beyond motion $(\mathrm{t}(28)=.45, p=.65$, Cohen's $\mathrm{d}=.16)$, similarly to the AUC analysis. These results can be seen in Figure 3. Hit and false alarm rates are reported in Table 1, and suggest that the motion contribution may be driven by lower false alarms when performing recognition in videos as compared to static images alone. 


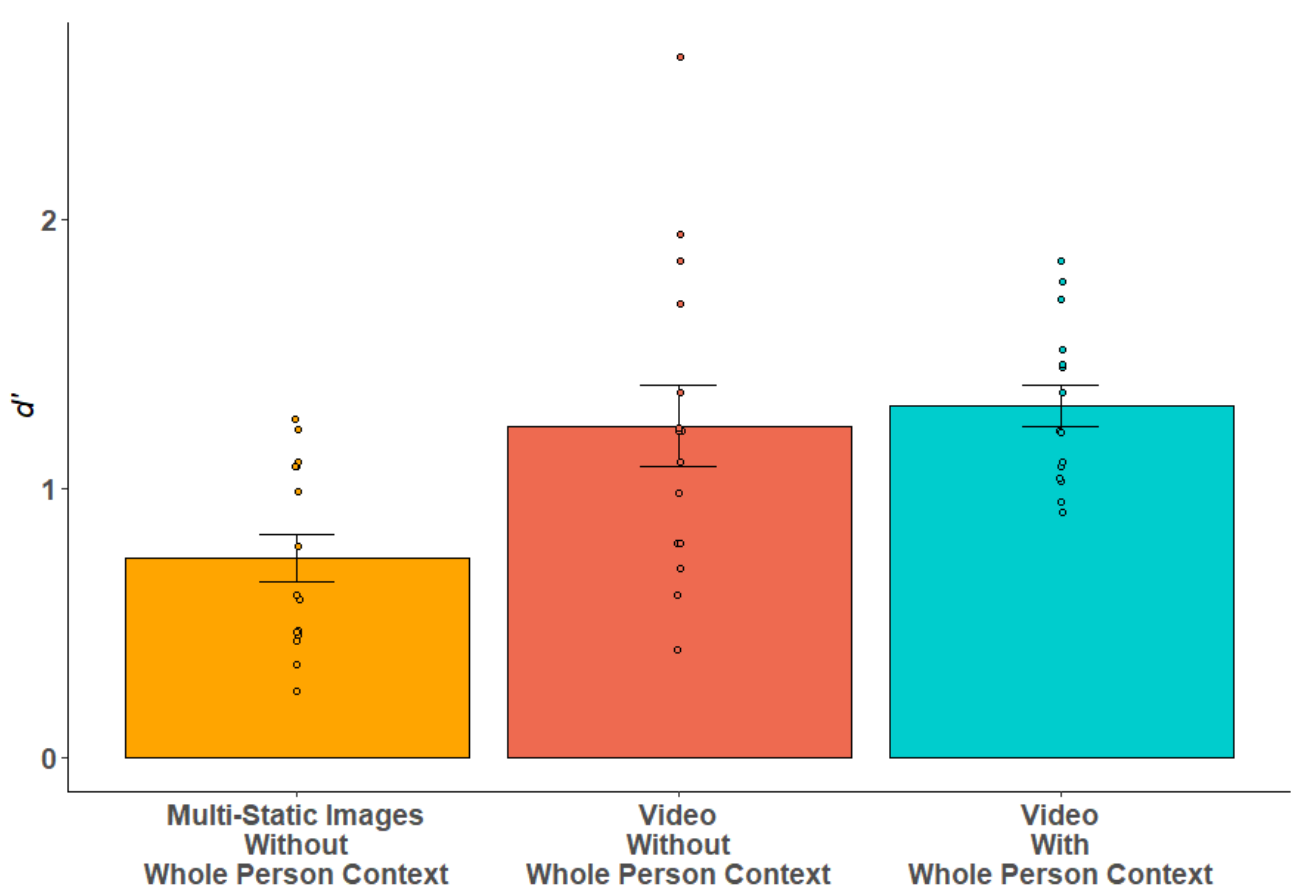

Figure 3. The $d^{\prime}$ in the three test conditions in Experiment 1. Error bars represent SEM, and points mark the scores of different participants within each test condition.

We further assessed possible differences in RTs on correct trials, confidence ratings on correct trials and the criterion of response between the conditions in our experiment. A one-way ANOVA examining the three recognition conditions revealed no effect of condition in the analysis of RTs $\left(F(2,42)=2.44, p=.10, \eta_{p}^{2}=.10\right)$, confidence ratings $(F(2,42)<1)$ or the criterion of response $(F(2,42)<1)$. These values can be seen in Table 1. 
Table 1

Experiment 1 Results

\begin{tabular}{lllll}
\hline Measure & Multi-Static Images & & Video \\
\cline { 2 - 2 } \cline { 5 - 5 } & $\begin{array}{l}\text { Without Whole Person } \\
\text { Context }\end{array}$ & & $\begin{array}{l}\text { Without Whole } \\
\text { Person Context }\end{array}$ & $\begin{array}{l}\text { With Whole } \\
\text { Person Context }\end{array}$ \\
\hline AUC & $.65(.02)$ & & $.76(.02)$ & $.79(.02)$ \\
$d^{\prime}$ & $.74(.09)$ & $1.23(.15)$ & $1.31(.08)$ \\
Hits & $.71(.03)$ & $.75(.04)$ & $.78(.03)$ \\
False Alarms & $.45(.03)$ & $.34(.04)$ & $.33(.03)$ \\
RT & $1.17(.09)$ & $1.42(.14)$ & $1.12(.07)$ \\
Confidence & $3.24(.11)$ & $3.11(.15)$ & $3.31(.10)$ \\
C & $-.23(.09)$ & $-.13(.11)$ & $-.19(.08)$ \\
\hline
\end{tabular}

Note. The mean AUC values, $d^{\prime}$, hit rates, false alarms rates, response times (RT), confidence and criterion (C) ratings in each of the experimental conditions in Experiment 1. RTs and confidence ratings are reported for correct trials only. SEM is reported for each measure in parenthesis.

\subsection{Discussion}

In Experiment 1 we examined the direct contribution of dynamic identity signatures from body motion to body-based person recognition. Recognizing identities from videos as compared to static images alone significantly improved body-based person recognition. This advantage is especially important since, using a very similar design to examine whole person recognition, Simhi \& Yovel, 2016 revealed no advantage to recognition in videos as compared to recognition from a single static image, in whole person recognition. Dynamic identity signatures can therefore contribute differently to body-based and whole person recognition, depending on the information available, as suggested in Yovel \& O’Toole, 2016. These findings also add to previous studies that show the importance of body motion to person recognition in particular when facial information is unclear, which was previously assessed in examining person recognition from a 
distance (Hahn et al., 2015; Simhi \& Yovel, 2019). However, these studies could not assess the role of body motion directly, either because the stimuli where presented only in videos (Hahn et al., 2015) or because whole person stimuli alone were shown, and therefore the specific contribution of the body could not be isolated (Simhi \& Yovel, 2019). Here we examined the contribution of motion to recognition from the body alone and the extent to which it can provide useful information for person recognition, when facial information is not available.

In addition to the contribution of dynamic identity signatures, we also examined the contribution of whole person context to dynamic body-based person recognition. We found that when performing body-based person recognition in video, whole person context did not contribute to person recognition beyond dynamic identity signatures. There are two possible explanations for this finding: first, it is possible that the videos of the body without whole person context used in this experiment did not completely abolish the perception of whole person context. The videos of the body without whole person context were created by covering the heads of the identities in the videos with rectangles that were colored in a hue that roughly matched the background behind the participants (as can be seen in Figure 1). These rectangles moved slightly throughout the video in order to cover the identities' heads in all of the video frames, according to their movement in the videos, and it is possible that the perception of a moving rectangle created the illusion of whole person context even though we strived to avoid it. Note that other methods for creating stimuli including the body alone, where a bag is sometimes used to cover the participants' heads for example (Rhodes, Jeffery, Boeing, \& Calder, 2013), or the face is obscured by a hat (Robbins \& Coltheart, 2015), do not avoid this problem as 
well, and the addition of a mono-color rectangle to a video makes every attempt to prevent the perception of a head like shape. Another possibility for the absence of an effect of context in this experiment is that seeing a body in motion may activate face processing mechanisms, in a similar manner as seeing whole person context does (as shown in static images in Brandman \& Yovel, 2010, 2012; Cox et al., 2004), and that the addition of actual head context contributes very little beyond this, a possibility which has yet to be assessed.

Thus, to further examine the contribution of whole person context to body-based person recognition we used static stimuli that enable a more direct and clear manipulation of body context. To create whole person context, a non-informative faceless head of an avatar was attached to the body, replacing the original head. To remove whole person context, the head was completely removed (see Figure 4). A similar manipulation, attaching an uninformative body to a face or completely removing it, was performed in order to examine the effect of whole person context on face-based person recognition, in the same experiment.

\section{Experiment 2: The effect of whole person context on static body-based and face-based person recognition}

In Experiment 2, we examined the contribution of whole person context in person recognition from static stimuli. In this experiment, in addition to examining body-based person recognition we also examined face-based person recognition. While approaching bodies were found to contribute to face-based person recognition as compared to static bodies (Pilz et al., 2011), static face-based person recognition with and without whole person context has not been directly examined. To test the effect of whole person context on body-based and face-based person 
recognition, four different test conditions, composed of different types of static images, were presented (see Figure 4): This included images of the body alone with and without whole person context, which allowed us to examine the contribution of whole person context to body-based person recognition, as well as images of the face alone with and without whole person context, which allowed us to examine the contribution of whole person context to face-based person recognition.

\subsection{Methods}

\subsubsection{Participants}

60 participants took part in this study (mean age $=23.08, S D=2.82,41$ female). Participants were randomly allocated to four different between participant conditions, each containing 15 participants. Participants were recruited at Tel Aviv University and participated in the study for

either course credit or payment. All participants had normal or corrected to normal vision and gave their informed consent to participate in the study by signing the appropriate consent form approved by the Tel Aviv University ethics committee.

\subsubsection{Stimuli}

The study stimuli in Experiment 2 were identical to those in Experiment 1. The test stimuli in each stimulus pair in Experiment 2 were edited static images extracted from one of the final frames of the second stimulus filmed for each identity, in which the full body was still visible. The background was removed in all of these images, which were then edited to create four different, 
matched conditions examining body and face-based person recognition, with and without whole person context, as detailed below (examples of these stimuli can be seen in Figure 4):

Body-based person recognition:

I. Body images without whole person context - in these stimuli the original face of the identity and most of the neck was completely removed so that the body only was visible. Note that for some of the female identities in the experiment who had long hair, the hair could be seen in these images as well.

II. Static Body with whole person context - these stimuli were created by taking the body only stimuli described above and attaching uninformative faceless heads to them. One male and one female faceless head of an avatar were extracted from Poser 7 (Smith Micro Software). The same male head was attached to all of the male bodies in the experiment, after adapting its size and slightly adjusting the color to fit as naturally as possible with the body. The same female head was attached to all female bodies in the experiment in the same manner. The heads therefore had no identifying information and had no relation to the bodies themselves. These heads were used solely in order to create the perception of whole person context, even though identification could in fact be made based on the body alone. The size of the body in these stimuli was matched to the size of the body in the body images without whole person context.

Face-based person recognition: 
III. Face images without whole person context - in these images the original body of the identity was removed from the neck down, so that only the face was visible.

IV. Face images with whole person context - these stimuli were created by attaching uninformative bodies to the face only images, in order to create the perception of whole person context. The bodies were extracted from Poser 7, and in the same manner used to create the body images with whole person context, only one male and one female body were extracted. These bodies were attached to the faces, according to their gender, and their size and color were slightly modified for each image in order to create as naturalistic an appearance as possible. The size of the face in these stimuli was matched to the size of the face in the face images without whole person context. Again, it should be noted that only the face contained informative identity related information in these stimuli. 


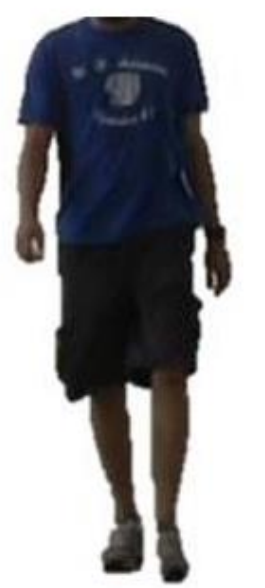

Without Whole Person Context

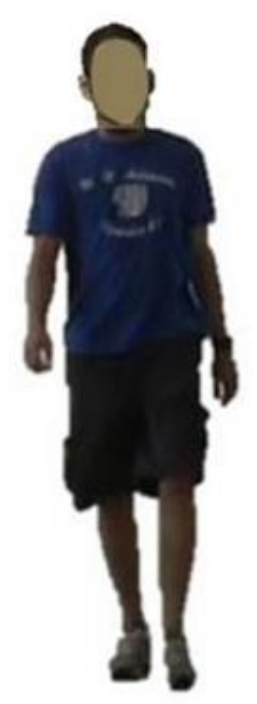

With Whole Person Context

Body-Based Person

Recognition
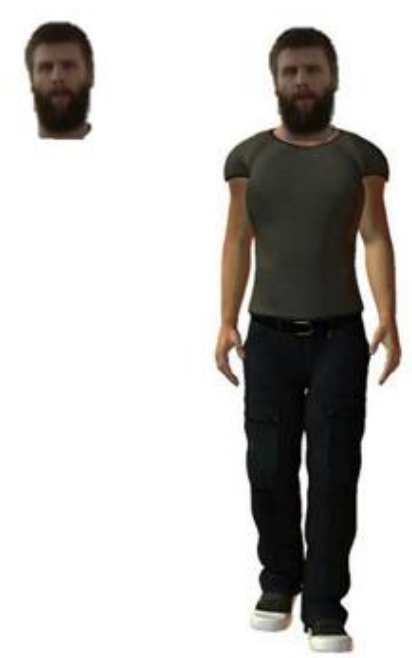

Without Whole

With Whole

Person Context

Face-Based Person

Recognition

Figure 4. Examples of the test stimuli in the four experimental condition in Experiment 2. These stimuli were used to examine the contribution of whole person context to body-based (left two images) and face-based (right two images) person recognition. The same identity is depicted in all of four stimuli.

\subsubsection{Design}

The design of Experiment 2 was identical to that in Experiment 1, with the only difference being in the test stimuli type and presentation. The test stimulus in each pair of stimuli varied between participants according to the experimental condition and could be an image of either the body without whole person context, the body with whole person context, the face without whole person context or the face with whole person context (see Figure 4). 


\subsubsection{Apparatus and Procedure}

The apparatus and procedure in this experiment were very similar to that in Experiment 1 and differed mainly in the test stimulus presentation. The test stimuli in Experiment 2 consisted of static images (in four experimental conditions, as detailed above), which were presented on the screen until participants made their recognition response. In addition, during the practice trial in Experiment 2, in the conditions which included whole person context participants were explicitly informed that the same heads (in body-based person recognition) or bodies (in facebased person recognition) would be used throughout the experiment, and that they were therefore uninformative for person recognition.

\subsubsection{Data Analysis}

Data analysis was performed in the same manner as in Experiment 1.

\subsection{Results}

In order to assess the participants' discrimination ability in body and face-based person recognition, with and without whole person context, we first plotted ROC curves for each of the four conditions in our experiment. These curves can be seen in Figure 5.

From the ROC curves we can see that body-based person recognition is extremely poor without whole person context, and does not differ from chance performance. When whole person context is available however, person recognition improves, even though it remains low overall. This contribution does not take place in face-based person recognition, which is similar with and without whole person context. 
In order to statistically examine these effects, we extracted the AUC from the ROC curves. The AUC values can be seen in Figure 5 (B) and Table 2. We performed a 2X2 Factorial ANOVA on the AUC values, examining recognition type (body/face-based) and whole person context (with/without). This analysis revealed a main effect of recognition type $(F(1,56)=282.53, p<$ $\left..001, \eta_{p}^{2}=.84\right)$, a main effect of whole person context $\left(F(1,56)=7.13, p=.01, \eta^{2}=.11\right)$ and an interaction $\left(F(1,56)=4.94, p=.03, \eta_{p}^{2}=.08\right)$. Post hoc Tukey analysis of this interaction revealed that it was due to a contribution of whole person context in body-based person recognition ( $p=$ $.006)$ but not face-based person recognition $(p=.99)$. Note that the lack of an effect in face-based person recognition was not due to a ceiling effect, with AUC values averaging at $.85-.86$ (SEM = $.01-.02)$ in face-based recognition conditions, as can be seen in Table 2. 


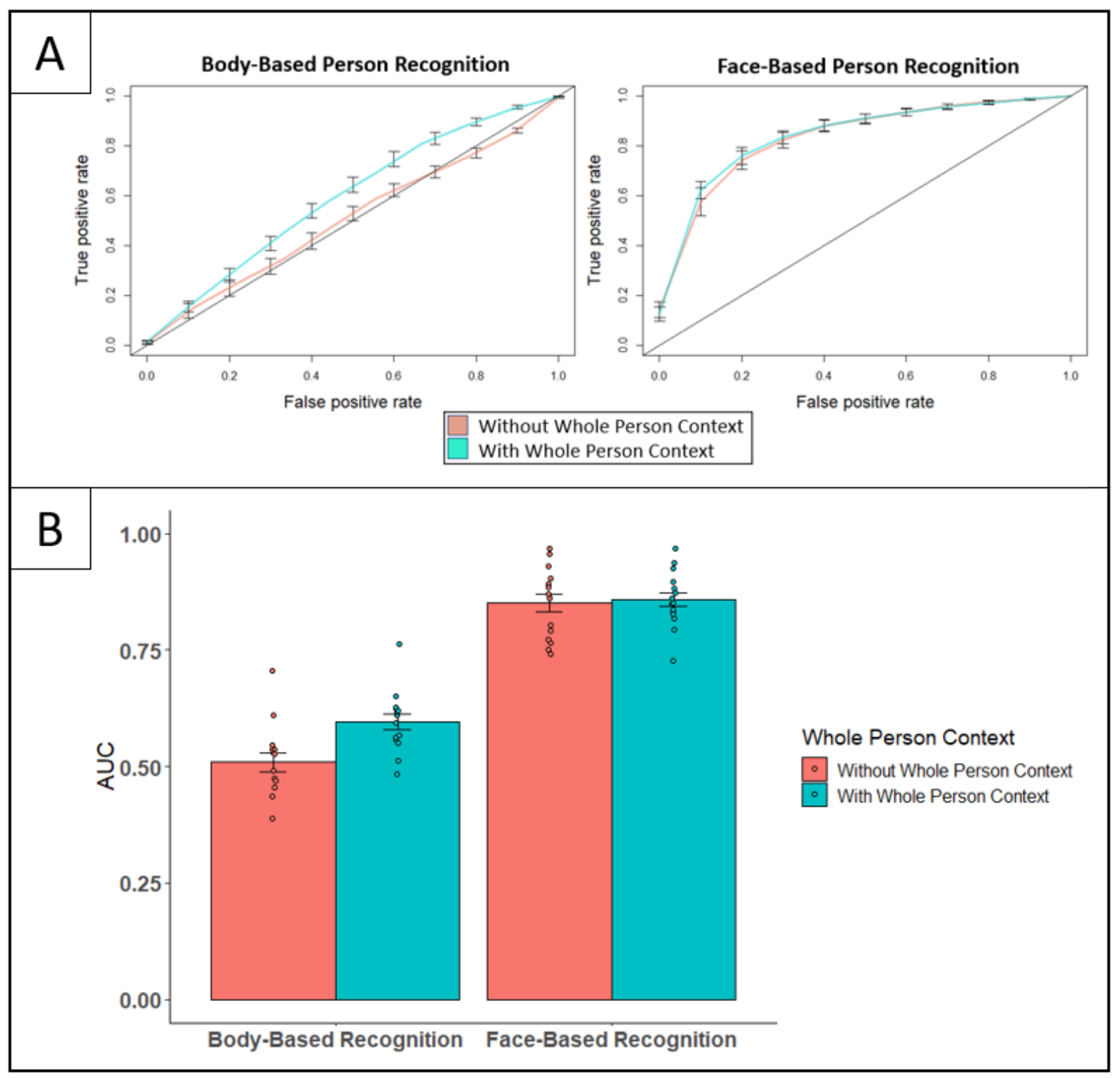

Figure 5. ROC curves (A) and AUC (B) depicting body and face-based person recognition, with and without whole person context. These results showed a whole person context effect for bodybased but not face-based person recognition. Error bars represent SEM for both panels, and points in Panel B mark the scores of different participants within each test condition.

As in Experiment 1, we also examined the $d^{\prime}$ of person recognition in the different conditions in our experiment. Similarly to the AUC analysis, we again found a main effect of 
recognition type $\left(F(1,56)=120.68, p<.001, \eta^{2} p=.68\right)$ due to greater discrimination in the facebased as compared to the body-based recognition conditions. In this case, however, there was no main effect of whole person context $\left(F(1,56)=2.02, p=.16, \eta_{p}^{2}=.03\right)$ or interaction $(F(1,56)$ <1). These results can be seen in Figure 6 and Table 2.

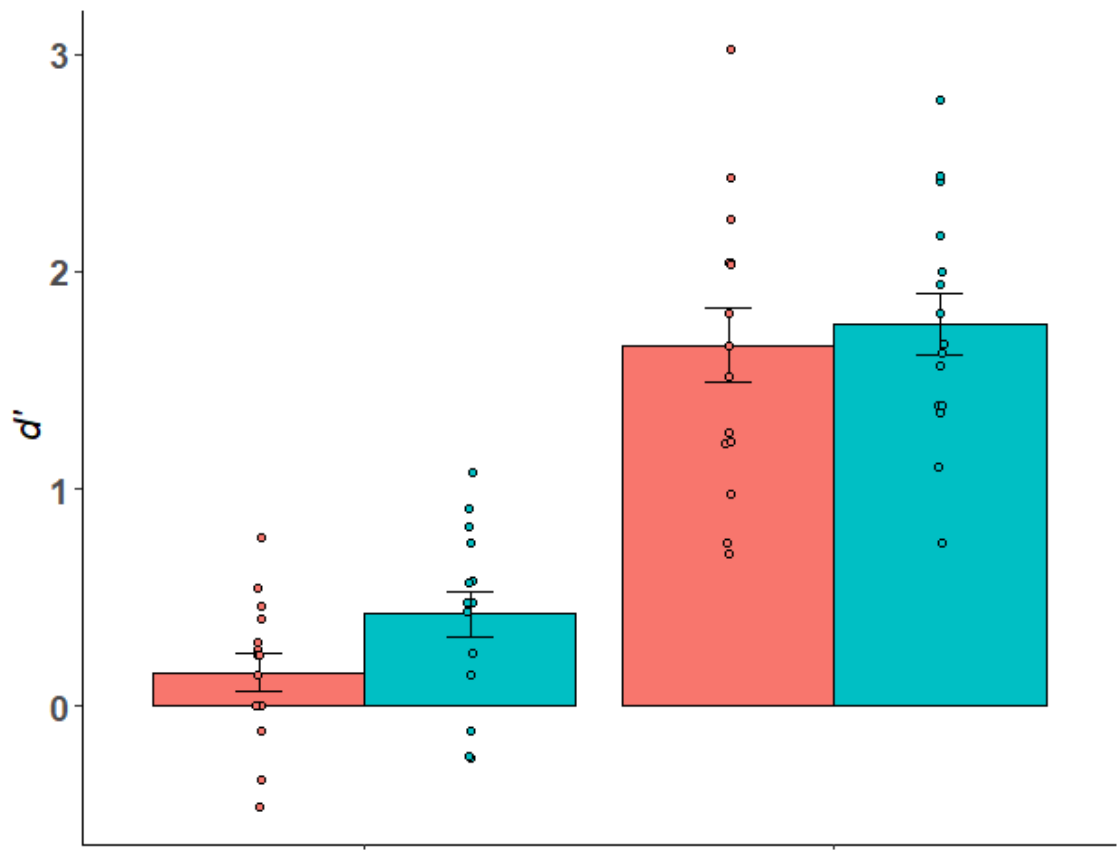

Whole Person Context

- Without Whole Person Context With Whole Person Context

Body-Based Recognition Face-Based Recognition

Figure 6. The $d^{\prime}$ in body and face-based person recognition, with and without whole person context. Error bars represent SEM, and points mark the scores of different participants within each test condition.

In addition, we examined RTs, confidence rating and the criterion of response $(C)$, in order to assess other possible effects of whole person context in body and face-based person recognition. In examining RTs, we again found a main effect of recognition type $(F(1,56)=8.95$, 
$p=.004, \eta_{p}^{2}=.14$ ) (due to longer RTs in the body-based recognition condition as compared to the face-based recognition condition) but no main effect of whole person context $(F(1,56)<1)$ or interaction $(F(1,56)<1)$.

In examining confidence ratings we also found a main effect of recognition type $(F(1,56)$ $=13.65, p<.001, \eta_{p}^{2}=.20$ ) (due to higher confidence in the face-based recognition condition as compared to the body-based recognition condition) but no main effect of whole person context $(F(1,56)<1)$ or interaction $(F(1,56)<1)$.

Finally, when examining the criterion of response we found no main effect of recognition type $\left(F(1,56)=2.54, p=.12, \eta_{p}^{2}=.04\right)$ and no main effect of whole person context $(F(1,56)=3.12$, $\left.p=.08, \eta_{p}^{2}=.05\right)$ or interaction $(F(1,56)<1)$.

These results can be found in Table 2 as well.

Table 2

Experiment 2 Results

\begin{tabular}{llllll}
\hline \multirow{2}{*}{ Measure } & \multicolumn{2}{l}{ Body-based Recognition } & & \multicolumn{2}{c}{ Face-based Recognition } \\
\cline { 2 - 3 } \cline { 5 - 6 } & Without Whole & With Whole & & Without Whole & With Whole \\
& Person Context & Person Context & & Person Context & Person Context \\
\hline AUC & $.51(.02)$ & $.60(.02)$ & & $.85(.02)$ & $.86(.01)$ \\
$d^{\prime}$ & $.15(.09)$ & $.42(.10)$ & & $1.66(.17)$ & $1.76(.14)$ \\
Hits & $.58(.03)$ & $.59(.04)$ & & $.79(.03)$ & $.75(.03)$ \\
False Alarms & $.52(.04)$ & $.44(.03)$ & & $.24(.03)$ & $.18(.03)$ \\
RT & $2.81(.18)$ & $2.71(.21)$ & & $2.14(.15)$ & $2.34(.15)$ \\
Confidence & $3.26(.07)$ & $3.38(.11)$ & & $3.74(.12)$ & $3.66(.10)$ \\
C & $-.15(.09)$ & $-.04(.08)$ & & $-.06(.08)$ & $.15(.10)$ \\
\hline
\end{tabular}


Note. The mean AUC values, $d^{\prime}$, hit rates, false alarms rates, response times (RT), confidence and criterion (C) ratings in each of the experimental conditions in Experiment 2. RTs and confidence ratings are reported for correct trials only. SEM is reported for each measure in parenthesis.

\subsection{Discussion}

In Experiment 2 we demonstrated that whole person context contributes to body-based but not face-based person recognition. In addition, we found that person recognition based on the body alone, without whole person context, is extremely poor and in fact does not differ from chance performance. These findings are especially important in light of the fact that most of the studies examining body-based person recognition to date have used conditions in which some form of whole person context may have been available. These findings suggest that this context may play a critical role in person recognition. Faceless heads attached to bodies have been shown to activate face processing mechanisms (Brandman \& Yovel, 2010, 2012; Cox et al., 2004), and these findings suggest that the activations of these mechanisms may mediate body processing. It should be kept in mind that in naturalistic situations, while we do see images of faces alone, it is rare that we are tasked with recognizing people based on the body alone. In naturalistic situations body-based person recognition is most likely to occur when seeing a person at a distance, where facial features are not clearly visible while the body shape is - so in fact in naturalistic situations, when we perform body-based person recognition we are most likely doing so in whole person context. The findings presented here support the notion that this context is necessary for body-based recognition to take place. 
The fact that no contribution of whole person context was found in this study for facebased person recognition is somewhat surprising. Pilz et al., 2011 examined face-based person recognition and demonstrated that approaching, uninformative bodies improve face-based person recognition as compared to static bodies by enabling faster recognition, however Pilz et al., 2011 did not directly compare face-based person recognition with and without whole person context in order to isolate the possible contribution of context from that of approaching motion. In addition, it has been shown that body context activates face-based processing mechanisms even without the presence of a face (Brandman \& Yovel, 2010, 2012; Cox et al., 2004), suggesting that face-based recognition may improve in whole person context. Nonetheless, our study showed that while the presence of a body may affect face processing mechanisms, when facial features are available a static body did not improve face-based person recognition.

Finally, this study also directly compares face and body-based person recognition. In line with the findings in O'Toole et al., 2011, using a different design we also find strong differences in face and body-based person recognition. Face-based person recognition is significantly more accurate than body-based person recognition and participants also responded faster and with greater confidence in face-based recognition. The results presented here stress that while identifying information is present in the body form, body-based person recognition is relatively poor and depends on additional factors, such as whole person context and the availability of dynamic identity signatures. 


\section{General Discussion}

In this study we revealed novel findings regarding the use of the body in person recognition. While many studies examining person recognition presented the face alone, relatively few studies have examined whole person recognition, and fewer studies yet have attempted to clarify what is the contribution of the body alone to person recognition. Here we revealed that body-based person recognition is poor but can be enhanced by dynamic identity signatures from body motion and whole person context.

In Experiment 1, we show that dynamic identity signatures from body motion play an important role in body-based person recognition. The findings of this study are especially important since they highlight differences between whole person recognition and body-based person recognition; Simhi \& Yovel, 2016, which used a similar design, did not reveal a contribution of dynamic identity signatures when recognizing the whole person, in contrast to the contribution found here for recognition from the body alone. Taken together, these findings suggest that dynamic identity signatures play a crucial role in body-based person recognition. However, they are most likely to be used in whole person recognition only under difficult recognition conditions, such as when people are seen from a distance or when facial information is not available (thus supporting and expanding upon the findings in Hahn et al., 2015 and O'Toole et al., 2011). In such cases, person recognition may depend more strongly on the body (Yovel \& O'Toole, 2016). Experiment 1 also examined the role of whole person context and showed no effect of the presence of the head on dynamic body-based person recognition. Nonetheless, this result could indicate that whole person context does not contribute to body-based person 
recognition beyond dynamic identity signatures, or that the body only videos presented in Experiment 1 failed to completely eliminate the perception of whole person context.

Therefore, in Experiment 2, we examined the contribution of whole person context in body and face-based recognition from static images, where whole person context can be manipulated more clearly. We show that whole person context plays an important role in bodybased person recognition from static images, where we were able to completely remove whole person context. Performance was at chance when recognizing identities from the body alone, however adding uninformative faceless heads in order to create whole person context significantly improved performance. It is important to stress that the faceless heads had no connection to the identities' bodies, and participants were informed in advance that the same heads would be presented during the experiment. Thus, the contribution of whole person context to body-based person recognition may be automatic. Previous studies examining the importance of body context in face perception have shown that when whole person context is available face processing mechanisms are activated, even when no faces are presented (Brandman \& Yovel, 2010, 2012; Cox et al., 2004). In daily life, we almost never perceive a person without some face context, and it is possible that the activation of face processing mechanisms as a result of this context presentation, contributes to body-based processing. Interestingly, no contribution of whole person context was found in face-based person recognition, indicating that while body processing mechanisms may depend on face processing mechanisms to some degree, the opposite dependency does not take place and static face-based person recognition does not improve when whole person context is available. 
Overall, this study highlights the importance of examining body-based person recognition. As demonstrated here, body-based person recognition is affected by unique processes, which differ from face-based person recognition and even whole person recognition. In daily life, we perceive both the body and the face, and it is therefore crucial to understand what influences face and body-based person recognition, and under what circumstances such types of recognition are performed.

\section{Acknowledgments}

We would like to thank Alice O'Toole for providing us with the Video Database of Moving Faces and People. Special thanks to undergraduate student Or Raybi and to Dr. Michal Berenstein-Eliav for their assistance in collecting the data for these experiments. The study was funded by an Israeli Science Foundation grant (446/2016) to GY.

\section{References}

Bindemann, M., Scheepers, C., Ferguson, H. J., \& Burton, A. M. (2010). Face, body, and center of gravity mediate person detection in natural scenes. Journal of Experimental Psychology. Human Perception and Performance, 36(6), 1477-1485. https://doi.org/10.1037/a0019057

Brainard, D. H. (1997). The Psychophysics Toolbox. Spatial Vision, 10(4), 433-436. https://doi.org/10.1163/156856897X00357

Brandman, T., \& Yovel, G. (2010). The body inversion effect is mediated by face-selective, not body- 
selective, mechanisms. The Journal of Neuroscience: The Official Journal of the Society for Neuroscience, 30(31), 10534-10540. https://doi.org/10.1523/JNEUROSCI.0911-10.2010

Brandman, T., \& Yovel, G. (2012). A face inversion effect without a face. Cognition, 125(3), 365-372. https://doi.org/10.1016/j.cognition.2012.08.001

Cox, D., Meyers, E., \& Sinha, P. (2004). Contextually evoked object-specific responses in human visual cortex. Science (New York, N.Y.), 304(5667), 115-117. https://doi.org/10.1126/science.1093110

Downing, P. E., Jiang, Y., Shuman, M., \& Kanwisher, N. (2001). A cortical area selective for visual processing of the human body. Science (New York, N.Y.), 293(5539), 2470-2473. https://doi.org/10.1126/science.1063414

Hahn, C. A., O'Toole, A. J., \& Phillips, P. J. (2015). Dissecting the time course of person recognition in natural viewing environments. British Journal of Psychology (London, England: 1953), 1-18. https://doi.org/10.1111/bjop.12125

Kanwisher, N., McDermott, J., \& Chun, M. M. (1997). The fusiform face area: a module in human extrastriate cortex specialized for face perception. The Journal of Neuroscience : The Official Journal of the Society for Neuroscience, 17(11), 4302-4311. Retrieved from http://www.ncbi.nlm.nih.gov/pubmed/9151747

Kanwisher, N., \& Yovel, G. (2006). The fusiform face area: a cortical region specialized for the perception of faces. Philosophical Transactions of the Royal Society of London. Series B, Biological Sciences, 361(1476), 2109-2128. https://doi.org/10.1098/rstb.2006.1934

Kleiner, M., Brainard, D., Pelli, D., Ingling, A., Murray, R., \& Broussard, C. (2007). What's new in Psychtoolbox-3. Perception, 36(14), 1. 
O’Toole, A. J., Harms, J., Snow, S. L., Hurst, D. R., Pappas, M. R., Ayyad, J. H., \& Abdi, H. (2005). A video database of moving faces and people. IEEE Transactions on Pattern Analysis and Machine Intelligence, 27(5), 812-816. https://doi.org/10.1109/TPAMI.2005.90

O’Toole, A. J., Phillips, P. J., Weimer, S., Roark, D. A., Ayyad, J., Barwick, R., \& Dunlop, J. (2011). Recognizing people from dynamic and static faces and bodies: dissecting identity with a fusion approach. Vision Research, 51(1), 74-83. https://doi.org/10.1016/j.visres.2010.09.035

O'Toole, A. J., Roark, D. A., \& Abdi, H. (2002). Recognizing moving faces: a psychological and neural synthesis. Trends in Cognitive Sciences, 6(6), 261-266. https://doi.org/10.1016/S13646613(02)01908-3

Pashler, H. (2002). Stevens' Handbook of Experimental Psychology, Methodology in Experimental Psychology (Vol 4). John Wiley \& Sons.

Peelen, M. V, \& Downing, P. E. (2005). Selectivity for the human body in the fusiform gyrus. Journal of Neurophysiology, 93(1), 603-608. https://doi.org/10.1152/jn.00513.2004

Pelli, D. G. (1997). The VideoToolbox software for visual psychophysics: transforming numbers into movies. Spatial Vision, 10(4), 437-442. https://doi.org/10.1163/156856897X00366

Pilz, K. S., Vuong, Q. C., Bülthoff, H. H., \& Thornton, I. M. (2011). Walk this way: approaching bodies can influence the processing of faces. Cognition, 118(1), 17-31. https://doi.org/10.1016/j.cognition.2010.09.004

Rhodes, G., Jeffery, L., Boeing, A., \& Calder, A. J. (2013). Visual coding of human bodies: perceptual aftereffects reveal norm-based, opponent coding of body identity. Journal of Experimental Psychology. Human Perception and Performance, 39(2), 313-317. 
https://doi.org/10.1037/a0031568

Rice, A., Phillips, P. J., Natu, V., An, X., \& O’Toole, A. J. (2013). Unaware person recognition from the body when face identification fails. Psychological Science, 24(11), 2235-2243. https://doi.org/10.1177/0956797613492986

Robbins, R. A., \& Coltheart, M. (2015). The relative importance of heads, bodies, and movement to person recognition across development. Journal of Experimental Child Psychology, 138, 1-14. https://doi.org/10.1016/j.jecp.2015.04.006

Simhi, N., \& Yovel, G. (2016). The contribution of the body and motion to whole person recognition. Vision Research, 122, 12-20. https://doi.org/10.1016/j.visres.2016.02.003

Simhi, N., \& Yovel, G. (2017). The role of familiarization in dynamic person recognition. Visual Cognition, 25(4-6), 550-562. https://doi.org/10.1080/13506285.2017.1307298

Simhi, N., \& Yovel, G. (2019). Dissociating identity from gait : A virtual reality study of the role of dynamic identity signatures in person 1-37. https://doi.org/https://doi.org/10.31234/osf.io/39g5k

Yovel, G., \& O’Toole, A. J. (2016). Recognizing People in Motion. Trends in Cognitive Sciences, 20(5), 383395. https://doi.org/10.1016/j.tics.2016.02.005 